\title{
Risk Aversion in Modeling of Cap-and-Trade Mechanism and Optimal Design of Emission Markets
}

\author{
Paolo Falbo and Juri Hinz
}

\begin{abstract}
According to theoretical arguments, a properly designed emission trading system should help reaching pollution reduction at low social burden based on the theoretical work of environmental economists, cap-and-trade systems are put into operations all over the world. However, the practice from emissions trading yields a real stress test for the underlying theory and reveals a number of its weak points. This paper aims to fill the gap between general welfare concepts underlying understanding of liberalized market and specific issues of real-world emission market operation. In our work, we present a novel technique to analyze emission market equilibrium in order to address diverse questions in the setting of risk-averse market players. Our contribution significantly upgrades all existing models in this field, which neglect risk-aversion aspects at the cost of having a wide range of singularities in their conclusions, now resolved in our approach. Furthermore, we show both how the architecture of an environmental market can be optimized under the realistic assumption of risk-aversion.
\end{abstract}

Keywords Emission markets - Social burden • Environmental policy $\cdot$ Risk aversion $\cdot$ General equilibrium $\cdot$ Risk-neutral measure

This research was partially supported under Australian Research Council's Discovery Projects funding scheme (project number: DP13010335)

\section{P. Falbo}

Department of Economics and Management, University of Brescia,

Contrada S. Chiara, 50, 25122 Brescia, BS, Italy

e-mail: falbo@eco.unibs.it

J. Hinz $(\bowtie)$

Sydney - School of Mathematics, University of Technology,

P.O. Box 123, Broadway, Sydney, NSW 2007, Australia

e-mail: juri.hinz@uts.edu.au 


\section{Practice of the EU ETS}

A properly designed emission trading system should help reducing pollution reduction with low social burden.

In this paper we understand it as a burden to the society, caused by energy production. We assume that it can be measured in monetary units including both, the overall production costs and an appropriately quantified environmental impact of energy production.

Originated from this idea, and based on the theoretical work of environmental economists, cap-and-trade systems have been put into operations all over the world.

The problem of design optimization for emission trading schemes has been addressed in [4]. This work shows that, in general, a traditional architecture of environmental markets is far from being optimal, meaning that appropriate alterations may provide significant improvements in emission reduction performances at lower social burden. Such improvements can be achieved by extending a regulatory framework, which we address below as extended scheme.

Let us explain this.

In the traditional scheme, it is assumed that the administrator allocates a predetermined allowance number to the market and sets a compliance date at which a penalty must be paid for each unit of pollutant not covered by allowances. Hence, the policy maker can exercise merely two controls, the so-called cap (total amount of allowances allocated to the market) and the penalty size. In theory, a desired pollution reduction can be reached at some costs for the society by an appropriate choice of these parameters. However, in practice, there is not much flexibility, since the cap is motivated politically and the penalty is determined to provide enough incentives for the required pollution reduction. As a result, the performance of the traditional scheme could be very poor in terms of social burden for the achieved reduction.

In an extended scheme, the policy maker has much more influence. The regulator can tax or subsidize the production in terms of monetary units or in terms of emission certificates. These additional controls can be implemented in a technology-sensitive way. Doing so, the merit order of technologies can be changed significantly. On this account, emission savings, triggered by certificate prices, also become controllable. The work [4] illustrates that, by an appropriate choice of additional controls, the market can reach a targeted pollution reduction at much lower social burden.

Although these theoretical findings are sound, intuitive, and practically important, the optimization of environmental market architectures could not be brought to a level suitable for practical implementation. There are two reasons for this.

(1) The existing approach [4] is based on the unrealistic assumption that each of the market players is non-risk-averse in the sense that it realizes a linear utility function. This assumption is not conform with the modern view and creates a number of singularities in the model. A priori, it is not even clear which conclusions of this work do hold under risk-aversion. 
(2) Although the practical advantage of such market design optimization is obvious, policy makers hardly can use the theoretical findings of [4], because their quantitative assessment requires optimal control techniques whose numerics is difficult.

In this work, we address both issues, namely:

$\left(1^{\prime}\right)$ We assume a non-linear utility function for market agents and show several properties of the market equilibrium which make market design optimization possible. With this, our model is brought in line with standard economic theory and is appropriate for further developments. We also emphasize that, to capture risk-aversion, a completely new argumentation has been developed.

$\left(2^{\prime}\right)$ We provide our study in a one-period setting. Being accessible without optimal control techniques, the results become evident and potentially usable for a broad audience, including practitioners and decision makers.

The paper is organized as follows. Section 2 discusses the literature developed concerning markets of emission certificates. In Sect. 3 we introduce our equilibrium model. Section 4 deepens the analysis of the equilibrium. Section 5 studies the social optimality of the equilibrium and proves that it corresponds to the overall minimumcost policy under a risk-neutral probability distribution. Section 6 discusses some perspectives of optimal market design. The final Sect. 7 provides conclusions.

\section{Theory of Marketable Pollution Rights}

The efficiency properties of environmental markets have been first addressed in $[6,10]$, who first advocated the principle that the "environment" is a good that can not be "consumed" for free. In particular, Montgomery describes a system of tradable certificates issued by a public authority coupled with fixing a cap to the total emissions, and, doing so, to force polluting companies paying proportionally to the environmental damage generated by their production activity. An emission certificate is representative of the permission to emit a given quantity of pollutant without being penalized. Companies with low environmental impact can sell excess certificates and the resulting revenue represents a general incentive to reduce pollution. Montgomery shows that the equilibrium price for a certificate must be driven by the cost of the most virtuous company to abate its marginal unit of pollutant. The key result of his analysis is that such a system guarantees that the reduction of pollution is distributed among the companies efficiently, that is minimizing their total costs.

After the seminal analysis of Montgomery, which is based on a deterministic and static model, the following research has taken the direction to the stochastic and multi-period settings. A literature review on the research which has developed after Montgomery's work can be found in [14]. A common result shared by all the analyses developed so far is that cap-and-trade systems indeed represent the most efficient way to reduce and control the environmental damage generated by the industrial activity. 
Let us mention the contributions which are directly related to our analysis. A majority of relatively recent papers $[1-5,11,13]$ are related to equilibrium models, where risk-neutral individuals optimize the expected value of their profit or cost function. The hypothesis of risk-neutrality of the agents is gracefully assumed in those contributions, since it significantly simplifies the proof that environmental markets are efficient. Some papers have considered explicitly risk-averse decision makers. One of them, [9], develops a pricing model for the spot and derivative pricing of environmental certificates in a single-period economy. In [7], the authors also develop a (multi-period) equilibrium pricing model for contingent claims depending on environmental certificates, where risk-averse agents maximize the expected utility of their profit function.

\section{One-Period Equilibrium of Emission Market}

To explain the emission price mechanism, we present a market model where a finite number of agents, indexed by the set $I$, is confronted with abatement of pollution. The key assumptions are:

- We consider a trading scheme in isolation, within a time horizon $[0, T]$, without credit transfer from and to other markets. That is, unused emission allowances expire worthless.

- There is no production strategy adjustment within the compliance period $[0, T]$. This means that the agents schedule their production plans for the entire period $[0, T]$ at the beginning. Allowances can be traded twice: at time $t=0$ at the beginning and at time $t=T$ immediately before emission reports are surrendered to the regulator.

- For the sake of simplicity, we set the interest rate to zero.

- Each agent decides how much energy to produce and how many allowances to trade.

Note that this one-period model is best suited for our needs to explain the core mechanism of market operation and to discuss its properties. A generalization to a multi-period framework is possible, but it gives no additional insights related to the goal of this work.

The $i$ th agent is specified by the set $\Xi^{i}$ of feasible production plans for the generation of energy (electricity) within one time period from $t=0$ to $t=T$. Further, we consider the following mappings, defined on $\Xi^{i}$, for each agent $i \in I$ :

$$
\xi_{0}^{i} \mapsto V_{0}^{i}\left(\xi_{0}^{i}\right), C_{0}^{i}\left(\xi_{0}^{i}\right), E_{T}^{i}\left(\xi_{0}^{i}\right)
$$

with the interpretation that for production plan $\xi_{0}^{i} \in \Xi^{i}$, the values $V_{0}^{i}\left(\xi_{0}^{i}\right), C_{0}^{i}\left(\xi_{0}^{i}\right)$, and $E_{0}^{i}\left(\xi_{0}^{i}\right)$ stand for the total production volume, the total production costs, and the total carbon dioxide emission, respectively. 
Production: At time $t=0$, each agent $i \in I$ faces the energy demand $D_{0} \in \mathbb{R}_{+}$of the entire market, the realized electricity price $P_{0} \in \mathbb{R}_{+}$, and the emission allowance price $A_{0} \in \mathbb{R}_{+}$. Based on this information, each agent decides on its production plan $\xi_{0}^{i} \in \Xi^{i}$, where $\Xi^{i}$ is the set of feasible production plans. Given $\xi_{0}^{i} \in \Xi^{i}$, at time $T$, agent realizes the total production costs,

$$
C_{0}^{i}\left(\xi_{0}^{i}\right) \in \mathbb{R}
$$

the production volume

$$
V_{0}^{i}\left(\xi_{0}^{i}\right) \in \mathbb{R}
$$

and the total revenue, $P_{0} V_{0}^{i}\left(\xi_{0}^{i}\right)$, from the electricity sold.

Allowance allocation: We assume that the administrator allocates a pre-determined number $\gamma_{0}^{i} \in[0, \infty$ [ of allowances to each agent $i$.

So far, we have introduced deterministic quantities. Let us now turn to uncertainties modeled by random variables on the probability space $(\Omega, \mathscr{F}, \mathbb{P})$.

Emission from production: Following the production plan $\xi_{0}^{i}$, the total pollution of agent $i$ is expressed as $E_{T}^{i}\left(\xi_{0}^{i}\right)$.

Remark (Randomness in demand and production) The question of randomness in energy demand and production deserves a careful argumentation. The reader may be confused by the assumption that in our one-period modeling, the time unit may correspond to the entire compliance period (which suggests a rather long time), such that our assumption on deterministic demand and unflexible production schedule appears unrealistic. To ease understanding, one shall imagine an artificial emission market model for short time period, say one day until compliance. The point of our proposal is that the elements, the arguments and the techniques required to define the optimal production plan on a daily basis are the same of those required to identify the plan $\xi_{0}^{i}$ over a generalized period $[0, T]$. The value of this toy model is that it allows a straight-forward generalization to the multi-period situation. In our oneperiod modeling, we assume that the nominal energy demand $D_{0}$ is non-random and is observed at the time $t=0$ when production decisions are made. We also suppose that the production plan $\xi_{0}^{i}$ of each agent is deterministically scheduled at time $t=0$. This view is in line with the current practice in energy business, where a nominal energy production volume along with a detailed schedule of production units is planed non-randomly in advance. Of course, the realized energy consumption deviates from what has been predicted. However, based on our experience in energy markets, it does not make sense to include this random factor into equilibrium modeling, since all decisions are made on the basis of a non-random demand anticipation and non-random customer's requests for energy delivery. To maintain energy consumption fluctuations in real-time, diverse auxiliary mechanisms are used. They can be considered as purely technical measures (security of supply by reserve margins). For this reason, we believe that it is natural to assume that, although the energy 
demand $D_{0}$ is known and production plan $\xi_{0}^{i}$ is deterministically scheduled at time $t=0$, the total emission, associated with this production can not be predicted with certainty at time $t=0$ when the production and trading decisions are made. In fact, in practice the producers have to manage diverse source of randomness while following production, (demand fluctuation, outages of generators) which yields usually small but unpredictable deviations $N^{i}$ from the nominal emission $E_{0}^{i}\left(\xi_{0}^{i}\right)$ associated with production plan $\xi_{0}^{i}$. Thus, let us agree that $E_{T}^{i}\left(\xi_{0}^{i}\right)$ is modeled as a random variable given as a sum

$$
E_{T}^{i}\left(\xi_{0}^{i}\right)=E_{0}^{i}\left(\xi_{0}^{i}\right)+N^{i}, \quad \xi_{0}^{i} \in \Xi^{i}, i \in I
$$

with deterministic function

$$
\xi_{0}^{i}: \Xi^{i} \rightarrow \mathbb{R}, \quad \xi_{0}^{i} \mapsto E_{0}^{i}\left(\xi_{0}^{i}\right)
$$

describing the dependence of the nominal emission on the production plan and a random variable $N^{i}$ standing for the deviation from the nominal emission. Note that the random emission $E_{T}^{i}\left(\xi_{0}^{i}\right)$ will be the only source of uncertainty in our model.

To ease our analysis, let us agree on the natural assumption that for production schedules $\xi_{0}^{i} \in \Xi_{i} i \in I$ the total market emission $\sum_{i \in I} E_{T}^{i}\left(\xi_{0}^{i}\right)$ possesses no point masses:

$$
\mathbb{P}\left(\sum_{i \in I} E_{T}^{i}\left(\xi_{0}^{i}\right)=z\right)=0 \quad \text { for all } z \in \mathbb{R}
$$

Allowance trading: At times $t=0, T$ the allowance permits can be exchanged between agents by trading at the prices $A_{0}$ and $A_{T}$, respectively. Denote by $\vartheta_{0}^{i}, \vartheta_{T}^{i}$ the change at times $t=0, T$ of the allowance number held by agent $i \in I$. Such trading yields a revenue, which is

$$
-\vartheta_{0}^{i} A_{0}-\vartheta_{T}^{i} A_{T}
$$

Note that $\vartheta_{0}^{i}$ and $A_{0}$ are deterministic, whereas $\vartheta_{T}^{i}$ and $A_{T}$ are modeled as random variables. Observe that sales are described by negative values of $\vartheta_{0}^{i}, \vartheta_{T}^{i}$, therefore (5) is non-negative random variable, if permits are sold.

Penalty payment: As mentioned above, the penalty $\pi \in[0,+\infty[$ must be paid at maturity $T$ for each unit of pollutant not covered by allowances. Given the changes at times $t=0, T$ due to allowance trading, i.e. $\vartheta_{0}^{i}$ and $\vartheta_{T}^{i}$, the production $\xi_{0}^{i}$, and the total number $\gamma_{0}^{i}$ of allowances allocated to agent $i \in I$, the loss of agent $i$ due to potential penalty payment is given by

$$
\pi\left(E_{0}^{i}\left(\xi_{T}^{i}\right)-\vartheta_{0}^{i}-\vartheta_{T}^{i}-\gamma_{0}^{i}\right)^{+}
$$

Individual profit: In view of (1)-(6), the profit of agent $i \in I$ following trading and production strategy $\left(\vartheta^{i}, \xi^{i}\right)=\left(\vartheta_{0}^{i}, \vartheta_{T}^{i}, \xi_{0}^{i}\right)$ depends on the market prices $(A, P)=$ 
$\left(A_{0}, A_{T}, P_{0}\right)$ for allowances and energy and is given by

$$
\begin{aligned}
L^{A, P, i}\left(\vartheta^{i}, \xi^{i}\right)= & -\vartheta_{0}^{i} A_{0}-\vartheta_{T}^{i} A_{T}-C_{0}^{i}\left(\xi_{0}^{i}\right)+P_{0} V_{0}^{i}\left(\xi_{0}^{i}\right) \\
& -\pi\left(E_{T}^{i}\left(\xi_{0}^{i}\right)-\vartheta_{0}^{i}-\vartheta_{T}^{i}-\gamma_{0}^{i}\right)^{+} .
\end{aligned}
$$

Note that the individual profit could be negative.

Risk-aversion and rational behavior: Suppose that the risk attitudes of each agent $i \in I$ are described by a pre-specified strictly increasing utility function $U^{i}: \mathbb{R} \rightarrow \mathbb{R}$. With this, the rational behavior of the agent $i$ is targeted on the maximization of the functional

$$
\left(\vartheta^{i}, \xi^{i}\right) \mapsto \mathbb{E}\left(U^{i}\left(L^{A, P, i}\left(\vartheta^{i}, \xi^{i}\right)\right)\right)
$$

over all the possible trading and production strategies $\left(\vartheta^{i}, \xi^{i}\right)=\left(\vartheta_{0}^{i}, \vartheta_{T}^{i}, \xi_{0}^{i}\right)$.

Energy demand: Suppose that at time $t=0$ all agents observe the total energy demand, which is described by $D_{0} \in \mathbb{R}_{+}$. Let us agree that the demand must be covered.

Market equilibrium: Following standard apprehension, a realistic market state is described by the so-called equilibrium - a situation where all allowance prices, all allowance positions, and all production decisions are such that each agent is satisfied by its own policy and, at the same time, natural restrictions are fulfilled.

Definition 1 Given energy demand $D_{0} \in \mathbb{R}_{+}$, the prices $\left(A^{*}, P^{*}\right)=\left(A_{0}^{*}, A_{T}^{*}\right.$, $\left.P_{0}^{*}\right)$ are called equilibrium prices, if, for each agent $i \in I$, there exists a strategy $\left(\vartheta^{i *}, \xi^{i *}\right)=\left(\vartheta_{0}^{i *}, \vartheta_{T}^{i *}, \xi_{0}^{i *}\right)$ such that:

(i) the energy demand is covered

$$
\sum_{i \in I} V_{0}^{i}\left(\xi_{0}^{i *}\right)=D_{0}
$$

(ii) the emission certificates are in zero net supply

$$
\sum_{i \in I} \vartheta_{t}^{i *}=0 \quad \text { almost surely for } t=0 \text { and } t=T \text {, }
$$

(iii) each agent $i \in I$ is satisfied by its own policy in the sense that

$$
\mathbb{E}\left(U^{i}\left(L^{A^{*}, P^{*}, i}\left(\vartheta^{i *}, \xi^{i *}\right)\right)\right) \geq \mathbb{E}\left(U^{i}\left(L^{A^{*}, P^{*}, i}\left(\vartheta^{i}, \xi^{i}\right)\right)\right)
$$

holds for any alternative strategy $\left(\vartheta^{i}, \xi^{i}\right)$.

The main objective of this section is to prove that in the present model the electricity price formation is determined by the usual merit order arguments, where the effect of emission regulation causes emission allowance prices to enter the costs of production 
at the specific emission rate. This issue can be considered as the core mechanism of any cap-and-trade system, since including pollution costs into final product prices causes a change of the merit order of production technologies towards a cleaner production. To formulate this result, let us elaborate on the opportunity costs and introduce additional definitions.

Opportunity costs: In the economic literature, they stand for the forgone benefit from using a certain strategy compared to the next best alternative. For example, the opportunity costs of farming own land is the amount which could be obtained by renting the land to someone else. Let us explain how the opportunity costs necessarily lead to windfall profits.

When facing energy (electricity) generation, producers consider a profit, which could be potentially realized when, instead of production, unused emission allowances were sold to the market. For instance, if the price of the emission certificates is 12 $€$ per tonne of $\mathrm{CO} 2$ and the production of one Megawatt-hour (MWh) emits two tonnes of $\mathrm{CO} 2$ (say, using a coal-fired steam turbine), then the producer must decide between two strategies which are equivalent in terms of their emission certificate balance:

- produce and sell one MWh to the market,

- do not produce this MWh and sell allowances covering two tonnes of CO2.

In this situation, the opportunity costs of producing one MWh are $2 \times 12=24 €$. Obviously, the agent produces energy only if the first strategy is at least as profitable as the second one. Thereby, both the production and the opportunity costs must be considered in the formation of the electricity market price. Clearly, if the production costs of electricity are $30 €$ per MWh, then the energy will be produced only if its price covers both the production and the opportunity costs. Thus electricity can only be delivered at a price exceeding $30+2 \times 12=54 €$. That is, in order to trigger the electricity production, the opportunity costs must be added to the production costs.

In the scientific community, this phenomenon is well-known under the name of cost-pass-through. An empirical analysis, see [12] confirms that the strategy of costpass-through is currently followed by the European energy producers. Furthermore, the detailed investigation of mathematical market models shows that the cost-passthrough is the only possible strategy in the so-called equilibrium state of the market. This can be interpreted as follows: when behaving optimally, the energy producers must pass the allowance price on to the consumers. Note that the producer obtains a windfall profit of $24 €$ in anycase: if electricity price is higher than 54, by passingthrough the price of the certificates (that he has received for free); if the price is less than 54 , by selling 2 certificates at $12 €$ on the emission market.

More importantly, it turns out that the cost-pass-through is nothing but the core mechanism responsible for the emission savings. Namely, due to the opportunity costs, clean technologies appear cheaper than emission-intense production strategies. For instance, the alternative generation technology represented by a gas turbine, which yields energy at the price of $40 €$ and emits only one tonne of CO2, hardly competes with a coal-fired steam turbine under generic regime (without emissions regulation). Namely, if there is no regulatory framework, then the coal-fired steam 
turbine is scheduled first and the gas turbine has to wait until the energy demand can not be covered by coal-fired steam technologies. However, given an emission regulation, the opposite is true: say, if the allowance price is equal to $12 €$ per tonne of $\mathrm{CO} 2$ as above, then the gas technology appears cheaper, operating at full costs of $40+1 \times 12=52 €$. Thus, the gas turbine is scheduled first, followed by the coal-fired steam turbine, which runs only if the installed gas turbine capacity does not cover the energy demand.

In the next section, we will show that the only rational behavior in equilibrium is to pass the opportunity costs on to the consumers. For this, we require additional notions.

Definition 2 Consider a given energy amount $d \in \mathbb{R}_{+}$and a given allowance price $a \in \mathbb{R}_{+}$.

(i) Introduce the individual opportunity merit order costs of agent $i \in I$ as

$$
\mathscr{C}^{i}(d, a)=\inf \left\{C_{0}^{i}\left(\xi_{0}^{i}\right)+a E_{0}^{i}\left(\xi_{0}^{i}\right): \xi_{0}^{i} \in \Xi^{i}, \quad V_{0}^{i}\left(\xi_{0}^{i}\right) \geq d\right\}
$$

An individual production plan $\xi_{0}^{i} \in \Xi^{i}$ is called conform with opportunity costs at emission price $a \in \mathbb{R}_{+}$if

$$
\mathscr{C}^{i}\left(V_{0}^{i}\left(\xi_{0}^{i}\right), a\right)=C_{0}^{i}\left(\xi_{0}^{i}\right)+a E_{0}^{i}\left(\xi_{0}^{i}\right)
$$

that is to say $\xi_{0}^{i}$ is confirm if it minimizes the production and emission costs among all the alternative plans offering the same generation and given emission price $a$.

(ii) Introduce the cumulative opportunity merit order costs as

$$
\mathscr{C}(d, a)=\inf \left\{\sum_{i \in I}\left(C_{0}^{i}\left(\xi_{0}^{i}\right)+a E_{0}^{i}\left(\xi_{0}^{i}\right)\right): \xi_{0}^{i} \in \Xi^{i}, \quad i \in I, \quad \sum_{i \in I} V_{0}^{i}\left(\xi_{0}^{i}\right) \geq d\right\}
$$

The production plans $\xi_{0}^{i} \in \Xi^{i}, i \in I$, are called conform with opportunity costs at emission price $a \in \mathbb{R}_{+}$if

$$
\mathscr{C}\left(\sum_{i \in I} V_{0}^{i}\left(\xi_{0}^{i}\right), a\right)=\sum_{i \in I}\left(C_{0}^{i}\left(\xi_{0}^{i}\right)+a E_{0}^{i}\left(\xi_{0}^{i}\right)\right)
$$

(iii) Any price $p \in \mathbb{R}_{+}$with the property that

$$
-\mathscr{C}(\tilde{d}, a)+p \tilde{d} \leq-\mathscr{C}(d, a)+p d \text { for all } \tilde{d} \in \mathbb{R}_{+}
$$

is referred to as an opportunity merit order electricity price at $(d, a)$ and it can be understand as the marginal cost for the entire generation sector given the level of demand $d$ and emission price $a$. 


\section{Properties of Equilibrium}

With these definitions, we now show that, within any equilibrium, the production plans are always conform with opportunity costs. Furthermore, the equilibrium electricity price is always an opportunity merit order price.

Proposition 1 Given energy demand $D_{0}$, let $\left(A^{*}, P^{*}\right)=\left(A_{0}^{*}, A_{T}^{*}, P_{0}^{*}\right)$ be the equilibrium prices with the corresponding strategies $\left(\vartheta^{i *}, \xi^{i *}\right), i \in I$, then the following points hold:

(i) For each agent $i \in I$, the individual production plan $\xi_{0}^{i *}$ is conform with opportunity costs at emission price $A_{0}^{*}$ :

$$
\mathscr{C}^{i}\left(V_{0}^{i}\left(\xi_{0}^{i *}\right), A_{0}^{*}\right)=C_{0}^{i}\left(\xi_{0}^{i *}\right)+A_{0}^{*} E_{0}^{i}\left(\xi_{0}^{i *}\right)
$$

(ii) The market production schedule $\xi_{0}^{i *}, i \in I$, is conform with opportunity costs at emission price $A_{0}^{*}$ :

$$
\mathscr{C}\left(\sum_{i \in I} V_{0}^{i}\left(\xi_{0}^{i *}\right), A_{0}^{*}\right)=\sum_{i \in I}\left(C_{0}^{i}\left(\xi_{0}^{i *}\right)+A_{0}^{*} E_{0}^{i}\left(\xi_{0}^{i *}\right)\right)
$$

(iii) $P_{0}^{*}$ is an opportunity merit order price at $\left(\sum_{i \in I} V_{0}^{i}\left(\xi_{0}^{i *}\right), A_{0}^{*}\right)$.

The direct economic consequence of this mathematical result is that each individual will organize its own production strategy by scheduling power production units in an increasing price order. Thereby their variable costs (which include the opportunity costs of using the emission certificates) are considered. Within such a schedule, a demand $d$ is satisfied by gradually turning on the most economic plants, until a generation level matching $d$ is reached. Furthermore, the above proposition states that such schedule is reached not only on the individual level, but also for the entire market. Namely, an overall demand $d$ is satisfied by gradually turning on the most economic plants until reaching a production which covers the demand $d$. Such aggregate ordering is usually called merit order, for this reason we call $\mathscr{C}^{i}(d, a)$ and $\mathscr{C}(d, a)$ (agent's $i$ ) opportunity merit order costs and cumulative opportunity merit order costs, respectively.

It is worth noticing that the opportunity merit order electricity price as defined in (9) is equal to the marginal cost of generating electricity when the level of demand is $d$ given certificate price $a$. Coupling this property with the merit order production in the electricity sector, implies that the most expensive production in the plan $\xi_{0}^{i}$ will determine the marginal cost at demand level $d$ and emission price $a$. The opportunity merit order electricity price at $(d, a)$ is defined as the lowest price, which is able to trigger the required production level.

Proof (i) Consider the equilibrium strategy $\left(\vartheta_{0}^{i *}, \xi_{0}^{i *}\right)$ of agent $i \in I$. Assume that the agent deviates from this strategy following an alternative production plan $\xi_{0}^{i} \in \Xi^{i}$. However, to keep the same emission credit balance, the difference $E_{0}^{i}\left(\xi_{0}^{i}\right)-E_{0}^{i}\left(\xi_{0}^{i *}\right)$ 
is traded at the market in addition to $\vartheta_{0}^{i *}$. That is, we change the equilibrium trading strategy $\left(\vartheta_{0}^{i *}, \vartheta_{T}^{i *}\right)$ to an alternative trading strategy $\left(\vartheta_{0}^{i}, \vartheta_{T}^{i}\right)$ given by

$$
\vartheta_{0}^{i}=\vartheta_{0}^{i *}+E_{0}^{i}\left(\xi_{0}^{i}\right)-E_{0}^{i}\left(\xi_{0}^{i *}\right), \quad \vartheta_{T}^{i}=\vartheta_{T}^{i *} .
$$

Note that we have changed only the initial position, from $\vartheta_{0}^{i *}$ to $\vartheta_{0}^{i}$, whereas the final position is the same $\vartheta_{T}^{i}=\vartheta_{T}^{i *}$. A direct calculation shows that the profit of this alternative strategy $\left(\vartheta^{i}, \xi^{i}\right)=\left(\vartheta_{0}^{i}, \vartheta_{T}^{i}, \xi_{0}^{i}\right)$ can be written as

$$
L^{A^{*}, P^{*}, i}\left(\vartheta^{i}, \xi^{i}\right)=L^{A^{*}, P^{*}, i}\left(\vartheta^{i *}, \xi^{i *}\right)+R\left(\xi_{0}^{i}, \xi_{0}^{i *}\right),
$$

i.e. it differs form the original profit $L^{A^{*}, P^{*}, i}\left(\vartheta^{i *}, \xi^{i *}\right)$ by the amount

$$
R\left(\xi_{0}^{i}, \xi_{0}^{i *}\right)=P_{0}^{*}\left(V_{0}^{i}\left(\xi_{0}^{i}\right)-V_{0}^{i}\left(\xi_{0}^{i *}\right)\right)+\left(C_{0}^{i}\left(\xi_{0}^{i *}\right)-C_{0}^{i}\left(\xi_{0}^{i}\right)\right)+A_{0}^{*}\left(E_{0}^{i}\left(\xi_{0}^{i *}\right)-E_{0}^{i}\left(\xi_{0}^{i}\right)\right) .
$$

Note that $R\left(\xi_{0}^{i}, \xi_{0}^{i *}\right)$ can not be positive, since otherwise

$$
L^{A^{*}, P^{*}, i}\left(\vartheta^{i}, \xi^{i}\right)>L^{A^{*}, P^{*}, i}\left(\vartheta^{i *}, \xi^{i *}\right)
$$

would yield

$$
\mathbb{E}\left(U^{i}\left(L^{A^{*}, P^{*}, i}\left(\vartheta^{i}, \xi^{i}\right)\right)\right)>\mathbb{E}\left(U^{i}\left(L^{A^{*}, P^{*}, i}\left(\vartheta^{i *}, \xi^{i *}\right)\right)\right),
$$

thus contradicting the optimality of the equilibrium strategy $\left(\vartheta^{i *}, \xi^{i *}\right)$ (see (8)). Now, from $R\left(\xi_{0}^{i}, \xi_{0}^{i *}\right) \leq 0$ we conclude that

$$
-C_{0}^{i}\left(\xi_{0}^{i *}\right)-A_{0}^{*} E_{0}^{i}\left(\xi_{0}^{i *}\right)+P_{0}^{*} V_{0}^{i}\left(\xi_{0}^{i *}\right) \geq-C_{0}^{i}\left(\xi_{0}^{i}\right)-A_{0}^{*} E_{0}^{i}\left(\xi_{0}^{i}\right)+P_{0}^{*} V_{0}^{i}\left(\xi_{0}^{i}\right)
$$

for each $\xi_{0}^{i} \in \Xi^{i}$. With this, we conclude the desired assertion (10) as follows: any alternative production plan $\xi_{0}^{i}$ which produces an energy amount $V_{0}^{i}\left(\xi_{0}^{i}\right)$ at least equal to $V_{0}^{i}\left(\xi_{0}^{i *}\right)$ must satisfy

$$
C_{0}^{i}\left(\xi_{0}^{i *}\right)+A_{0}^{*} E_{0}^{i}\left(\xi_{0}^{i *}\right) \leq C_{0}^{i}\left(\xi_{0}^{i}\right)+A_{0}^{*} E_{0}^{i}\left(\xi_{0}^{i}\right) .
$$

Thus,

$$
C_{0}^{i}\left(\xi_{0}^{i *}\right)+A_{0}^{*} E_{0}^{i}\left(\xi_{0}^{i *}\right)=\inf \left\{C_{0}^{i}\left(\xi_{0}^{i}\right)+A_{0}^{*} E_{0}^{i}\left(\xi_{0}^{i}\right): \xi_{0}^{i} \in \Xi^{i}, \quad V_{0}^{i}\left(\xi_{0}^{i}\right) \geq V_{0}^{i}\left(\xi_{0}^{i *}\right)\right\} .
$$

(ii) Summing up (12) over $i \in I$, yields, for arbitrary choices of $\xi_{0}^{i} \in \Xi^{i}, i \in I$, 


$$
\begin{aligned}
-\sum_{i \in I} & \left(C_{0}^{i}\left(\xi_{0}^{i *}\right)+A_{0}^{*} E_{0}^{i}\left(\xi_{0}^{i *}\right)\right)+P_{0}^{*} \sum_{i \in I} V_{0}^{i}\left(\xi_{0}^{i *}\right) \\
& \geq-\sum_{i \in I}\left(C_{0}^{i}\left(\xi_{0}^{i}\right)+A_{0}^{*} E_{0}^{i}\left(\xi_{0}^{i}\right)\right)+P_{0}^{*} \sum_{i \in I} V_{0}^{i}\left(\xi_{0}^{i}\right) .
\end{aligned}
$$

From this, we conclude that, for any choice $\xi_{0}^{i} \in \Xi^{i}, i \in I$, of production plans satisfying

$$
\sum_{i \in I} V_{0}^{i}\left(\xi_{0}^{i}\right) \geq \sum_{i \in I} V_{0}^{i}\left(\xi_{0}^{i *}\right)
$$

it holds

$$
\sum_{i \in I}\left(C_{0}^{i}\left(\xi_{0}^{i *}\right)+A_{0}^{*} E_{0}^{i}\left(\xi_{0}^{i *}\right)\right) \leq \sum_{i \in I}\left(C_{0}^{i}\left(\xi_{0}^{i}\right)+A_{0}^{*} E_{0}^{i}\left(\xi_{0}^{i}\right)\right)
$$

implying the desired assertion (11).

(iii) We need to prove that, for any $\tilde{d} \in \mathbb{R}_{+}$,

$$
-\mathscr{C}\left(\tilde{d}, A_{0}^{*}\right)+P_{0}^{*} \tilde{d} \leq-\mathscr{C}\left(\sum_{i \in I} V_{0}^{i}\left(\xi_{0}^{i *}\right), A_{0}^{*}\right)+P_{0}^{*} \sum_{i \in I} V_{0}^{i}\left(\xi_{0}^{i *}\right) .
$$

For each choice of production strategies $\xi_{0}^{i} \in \Xi^{i}, i \in I$, estimate (13), combined with (11), gives

$$
\begin{aligned}
& -\mathscr{C}\left(\sum_{i \in I} V_{0}^{i}\left(\xi_{0}^{i *}\right), A_{0}^{*}\right)+P_{0}^{*} \sum_{i \in I} V_{0}^{i}\left(\xi_{0}^{i *}\right) \\
& \geq-\sum_{i \in I}\left(C_{0}^{i}\left(\xi_{0}^{i}\right)+A_{0}^{*} E_{0}^{i}\left(\xi_{0}^{i}\right)\right)+P_{0}^{*} \sum_{i \in I} V_{0}^{i}\left(\xi_{0}^{i}\right) .
\end{aligned}
$$

In particular, if the strategies are chosen from

$$
\left\{\left(\xi_{0}^{i}\right)_{i \in I}: \xi_{0}^{i} \in \Xi^{i}, \quad i \in I, \quad \sum_{i \in I} V_{0}^{i}\left(\xi_{0}^{i}\right) \geq \tilde{d}\right\}
$$

then it holds that

$$
-\mathscr{C}\left(\sum_{i \in I} V_{0}^{i}\left(\xi_{0}^{i *}\right), A_{0}^{*}\right)+P_{0}^{*} \sum_{i \in I} V_{0}^{i}\left(\xi_{0}^{i *}\right) \geq-\sum_{i \in I}\left(C_{0}^{i}\left(\xi_{0}^{i}\right)+A_{0}^{*} E_{0}^{i}\left(\xi_{0}^{i}\right)\right)+P_{0}^{*} \tilde{d} .
$$

Passing on the right-hand side of this inequality to

$$
\mathscr{C}\left(\tilde{d}, A_{0}^{*}\right):=\inf \left\{\sum_{i \in I}\left(C_{0}^{i}\left(\xi_{0}^{i}\right)+A_{0}^{*} E_{0}^{i}\left(\xi_{0}^{i}\right)\right): \xi_{0}^{i} \in \Xi^{i}, i \in I, \quad \sum_{i \in I} V_{0}^{i}\left(\xi_{0}^{i}\right) \geq \tilde{d}\right\},
$$


yields the desired assertion

$$
-\mathscr{C}\left(\sum_{i \in I} V_{0}^{i}\left(\xi_{0}^{i *}\right), A_{0}^{*}\right)+P_{0}^{*} \sum_{i \in I} V_{0}^{i}\left(\xi_{0}^{i *}\right) \geq-\mathscr{C}\left(\tilde{d}, A_{0}^{*}\right)+P_{0}^{*} \tilde{d}
$$

Remark The statement (ii) of the above proposition characterizes equilibrium in terms of aggregated quantities. Once the equilibrium is reached, the production schedule represents the cheapest way to satisfy the demand. From this perspective, the reader may conclude that the equilibrium production schedule can be obtained as a production plan which minimizes the overall costs among those which cover a given demand, indicating that only aggregated quantities do influence the equilibrium. However, we shall emphasize that the equilibrium still heavily depends on individual ingredients (such as initial endowments and risk aversion), which enter through the initial allowance price.

Now, we show another natural property of the equilibrium allowance prices. It turns out that there is no arbitrage allowance trading and that the terminal allowance price is digital.

Proposition 2 Given energy demand $D_{0}$, let $\left(A^{*}, P^{*}\right)=\left(A_{0}^{*}, A_{T}^{*}, P_{0}^{*}\right)$ be the equilibrium prices with the corresponding strategies $\left(\vartheta^{i *}, \xi^{i *}\right), i \in I$, . It holds:

(i) There exists a risk-neutral measure $\mathbb{Q}^{*} \sim \mathbb{P}$ such that $A^{*}=\left(A_{0}^{*}, A_{T}^{*}\right)$ follows a martingale with respect to $\mathbb{Q}^{*}$.

(ii) The terminal allowance price in equilibrium is digital

$$
A_{T}^{*}=\pi 1_{\left\{\sum_{i \in I} E_{T}^{i}\left(\xi_{0}^{i *}\right)-\gamma_{0} \geq 0\right\}} .
$$

Proof (i) According to the first fundamental theorem of asset pricing, see [8] in discrete-time setting, the existence of the so-called equivalent martingale measure satisfying $A_{0}^{*}=\mathbb{E}^{\mathbb{Q}^{*}}\left(A_{T}\right)$ is ensured by the absence of arbitrage. Fortunately, in our framework, the absence of arbitrage follows from the equilibrium notion, as we show next. We thus conclude (i) of the above theorem and it remains to verify that the equilibrium rules out all arbitrage opportunities for allowance trading. Let us follow an indirect proof, assuming that $v_{0}$ is an arbitrage allowance trading, meaning that

$$
\mathbb{P}\left(v_{0}\left(A_{T}^{*}-A_{0}^{*}\right) \geq 0\right)=1, \quad \mathbb{P}\left(v_{0}\left(A_{T}^{*}-A_{0}^{*}\right)>0\right)>0 .
$$

Based on this we obtain a contradiction by showing that each agent $i$ can change its own policy $\left(\vartheta^{i *}, \xi^{i *}\right)$ to an improved strategy $\left(\tilde{\vartheta}^{i}, \xi^{i *}\right)$ satisfying

$$
\mathbb{E}\left(U^{i}\left(L^{A^{*}, i}\left(\vartheta^{i *}, \xi^{i *}\right)\right)\right)<\mathbb{E}\left(U^{i}\left(L^{A^{*}, i}\left(\tilde{\vartheta}^{i}, \xi^{i *}\right)\right)\right) .
$$

The improvement is achieved by incorporating arbitrage $v_{0}$ into the allowance trading of each agent $i$ as follows:

$$
\tilde{\vartheta}_{0}^{i}:=\vartheta_{0}^{i *}+v_{0}, \quad \tilde{\vartheta}_{T}^{i}:=\vartheta_{T}^{i *}-v_{0}
$$


Indeed, the revenue improvement from allowance trading is

$$
-\tilde{\vartheta}_{0}^{i} A_{0}^{*}-\tilde{\vartheta}_{T}^{i} A_{T}^{*}=-\vartheta_{0}^{i} A_{0}^{*}-\vartheta_{T}^{i} A_{T}^{*}+v_{0}\left(A_{T}^{*}-A_{0}^{*}\right),
$$

which we combine with (15) to see that

$$
\mathbb{P}\left(L^{A, i}\left(\vartheta^{i *}, \xi^{i *}\right) \leq L^{A, i}\left(\tilde{\vartheta}^{i}, \xi^{i *}\right)\right)=1, \quad \mathbb{P}\left(L^{A, i}\left(\vartheta^{i *}, \xi^{i}\right)<L^{A, i}\left(\tilde{\vartheta}^{i}, \xi^{i *}\right)\right)>0,
$$

which implies (16), therefore contradicting the optimality of $\left(\vartheta^{i *}, \xi^{i *}\right)$.

(ii) From equilibrium property (8), it follows that for almost each $\omega \in \Omega$ the terminal allowance position adjustment $\vartheta_{T}(\omega)$ is a maximizer on $\mathbb{R}$ to

$$
z \mapsto-z A_{T}^{*}(\omega)-\pi\left(E_{T}^{i}\left(\xi_{0}^{i *}\right)(\omega)-\vartheta_{0}^{i *}-\gamma_{0}^{i}-z\right)^{+} .
$$

Note that a maximizer of this mapping exists only if $0 \leq A_{T}^{*}(\omega) \leq \pi$. That is, the terminal allowance price in equilibrium must be within the interval $A_{T}^{*} \in[0, \pi]$ almost surely. Let us show now that the price actually attains only boundary values almost surely, i.e.

$$
A_{T}^{*} \in\{0, \pi\} \quad \text { almost surely. }
$$

Suppose that an intermediate value $\left.A_{T}^{*}(\omega) \in\right] 0, \pi[$ is taken, then the unique maximizer of function (17) is attained on $E_{T}^{i}\left(\xi_{T}^{i *}\right)(\omega)-\vartheta_{0}^{i *}-\gamma_{0}^{i}$. This implies that $\vartheta_{T}^{i *}(\omega)=E_{T}^{i}\left(\xi_{0}^{i *}\right)(\omega)-\vartheta_{0}^{i *}-\gamma_{0}^{i}$ holds for each $i \in I$, and a summation over $i$ yields

$$
\sum_{i \in I} \vartheta_{T}^{i *}(\omega)=\sum_{i \in I}\left(E_{T}^{i}\left(\xi_{0}^{i *}\right)(\omega)-\vartheta_{0}^{i *}-\gamma_{0}^{i}\right)=\sum_{i \in I} E_{T}^{i}\left(\xi_{0}^{i *}\right)(\omega)-\gamma_{0}
$$

Note that equilibrium property (7) ensures that the random variable on the left-hand side of the above equality is zero almost surely. Thus, the inclusion

$$
\left\{\omega: A_{T}^{*} \in\right] 0, \pi[\} \subseteq\left\{\omega: \sum_{i \in I} E_{T}^{i}\left(\xi_{0}^{i *}\right)-\gamma_{0}=0\right\}
$$

holds almost surely. Because of (4), the probability of the event on the right-hand side of the above inclusion is zero, which shows (18).

If $A_{T}^{*}(\omega)=0$, then a maximizer $\vartheta_{T}^{i *}(\omega)$ to the function (17) is attained on $\left[E_{T}^{i}\left(\xi_{0}^{i *}\right)(\omega)-\vartheta_{0}^{i *}-\gamma_{0}^{i},+\infty[\right.$. Hence

$$
\left\{\omega: A_{T}^{*}=0\right\} \subseteq\left\{\omega: E_{T}^{i}\left(\xi_{0}^{i *}\right)-\vartheta_{0}^{i *}-\gamma_{0}^{i} \leq \vartheta_{T}^{i *}\right\}
$$

holds almost surely for each $i \in I$, which implies that 


$$
\left\{\omega: A_{T}^{*}=0\right\} \subseteq\left\{\omega: \sum_{i \in I} E_{T}^{i}\left(\xi_{0}^{i *}\right)-\gamma_{0} \leq \sum_{i \in I} \vartheta_{T}^{i *}\right\}
$$

holds almost surely. Now, because of the equilibrium property (7), we obtain the almost sure inclusion

$$
\left\{\omega: A_{T}^{*}=0\right\} \subseteq\left\{\omega: \sum_{i \in I} E_{T}^{i}\left(\xi_{0}^{i *}\right)-\gamma_{0} \leq 0\right\} .
$$

Since the probability of $\left.A_{T}^{*} \in\right] 0, \pi$ [ is zero (19), we conclude for the complementary event that

$$
\left\{\omega: A_{T}^{*}=\pi\right\} \supseteq\left\{\omega: \sum_{i \in I} E_{T}^{i}\left(\xi_{0}^{i *}\right)-\gamma_{0} \geq 0\right\}
$$

holds almost surely. Let us show the opposite inclusion. If $A_{T}^{*}(\omega)=\pi$, then a maximizer $\vartheta_{T}^{i *}(\omega)$ to function (17) is attained on $\left.]-\infty, E_{T}^{i}\left(\xi_{0}^{i *}\right)(\omega)-\vartheta_{0}^{i *}-\gamma_{0}^{i}\right]$. Hence,

$$
\left\{\omega: A_{T}^{*}=\pi\right\} \subseteq\left\{E_{T}^{i}\left(\xi_{0}^{i *}\right)-\vartheta_{0}^{i *}-\gamma_{0}^{i} \geq \vartheta_{T}^{i *}\right\}
$$

holds almost surely for each $i \in I$, which implies that

$$
\left\{\omega: A_{T}^{*}=\pi\right\} \subseteq\left\{\omega: \sum_{i \in I} E_{T}^{i}\left(\xi_{0}^{i *}\right)-\gamma_{0} \geq \sum_{i \in I} \vartheta_{T}^{i *}\right\}
$$

holds almost surely. Now, because of the equilibrium property (7), we obtain

$$
\left\{\omega: A_{T}^{*}=\pi\right\} \subseteq\left\{\omega: \sum_{i \in I} E_{T}^{i}\left(\xi_{0}^{i *}\right)-\gamma_{0} \geq 0\right\} .
$$

Finally, combine inclusions (20) and (21) to obtain assertion (14).

\section{Social Optimality}

To formulate social optimality, we require additional notations. Given production strategies $\xi_{0}^{i} \in \Xi^{i}, i \in I$, we denote the overall market production schedule by $\xi_{0}=\left(\xi_{0}^{i}\right)_{i \in I}$ and introduce the total production costs $C_{0}$, the total production volume $V_{0}$, and the total carbon dioxide emission $E_{T}$ and the total nominal carbon dioxide emission, defined by

$$
C_{0}\left(\xi_{0}\right)=\sum_{i \in I} C_{0}^{i}\left(\xi_{0}^{i}\right), \quad V\left(\xi_{0}\right)=\sum_{i \in I} V_{0}^{i}\left(\xi_{0}^{i}\right), \quad E_{T}\left(\xi_{0}\right)=\sum_{i \in I} E_{T}^{i}\left(\xi_{0}^{i}\right), \quad E_{0}\left(\xi_{0}\right)=\sum_{i \in I} E_{0}^{i}\left(\xi_{0}^{i}\right) .
$$


Having in mind that $C_{0}\left(\xi_{0}\right)$ stands for the overall costs of the production and interpreting $\pi\left(E_{T}\left(\xi_{0}\right)-\gamma_{0}\right)^{+}$as a proxy of the environmental impact of the production schedule $\xi_{0}$, let us agree that

$$
B\left(\xi_{0}\right)=C_{0}\left(\xi_{0}\right)+\pi\left(E_{T}\left(\xi_{0}\right)-\gamma_{0}\right)^{+}
$$

expresses the social burden caused by the overall production plan $\xi_{0} \in \times_{i \in I} \Xi^{i}$.

It turns out that the equilibrium strategy minimizes the social burden among all production strategies which cover a given demand.

Proposition 3 Given energy demand $D_{0}$, let $\left(A^{*}, P^{*}\right)=\left(A_{0}^{*}, A_{T}^{*}, P_{0}^{*}\right)$ be the equilibrium prices with the corresponding strategies $\left(\vartheta^{i *}, \xi^{i *}\right), i \in I$. Let $\mathbb{Q}^{*}$ be a risk-neutral measure whose existence is shown in Proposition 2. Then

$$
E_{0}^{\mathbb{Q}^{*}}\left(B\left(\xi_{0}^{*}\right)\right) \leq E_{0}^{\mathbb{Q}^{*}}\left(B\left(\xi_{0}\right)\right)
$$

holds for each production schedule $\xi_{0}=\left(\xi_{0}^{i}\right)_{i \in I} \in \times_{i \in I} \Xi^{i}$ which yields at least the same production volume, $V_{0}\left(\xi_{0}\right) \geq V_{0}\left(\xi_{0}^{*}\right)=D_{0}$.

Proof For each convex function $f: \mathbb{R} \rightarrow \mathbb{R}, x \mapsto f(x)$, it holds $f(x)+\nabla f(x) h \leq$ $f(x+h), h \in \mathbb{R}$, where $\nabla f(x)$ stands for one of the sub-gradients of $f$ at the point $x$. In particular, for convex function $f: \mathbb{R} \rightarrow \mathbb{R}_{+}, x \mapsto x^{+}$, we obtain $x^{+}+1_{\{x \geq 0\}} h \leq(x+h)^{+}$for all $x, h \in \mathbb{R}$. With the equilibrium production strategy $\xi_{0}^{*}=\left(\xi_{0}^{i *}\right)_{i \in I}$, we conclude that

$$
\left(E_{T}\left(\xi_{0}^{*}\right)-\gamma_{0}\right)^{+}+1_{\left\{E_{T}\left(\xi_{0}^{*}\right)-\gamma_{0} \geq 0\right\}}\left(E_{T}\left(\xi_{0}\right)-E_{T}\left(\xi_{0}^{*}\right)\right) \leq\left(E_{T}\left(\xi_{0}\right)-\gamma_{0}\right)^{+}
$$

holds almost surely for any production strategy $\xi_{0} \in \times_{i \in I} \Xi^{i}$. Using our model assumption (3) we deduce $E_{T}\left(\xi_{0}\right)-E_{T}\left(\xi_{0}^{*}\right)=E_{0}\left(\xi_{0}\right)-E_{0}\left(\xi_{0}^{*}\right)$ which gives

$$
\left(E_{T}\left(\xi_{0}^{*}\right)-\gamma_{0}\right)^{+}+1_{\left\{E_{T}\left(\xi_{0}^{*}\right)-\gamma_{0} \geq 0\right\}}\left(E_{0}\left(\xi_{0}\right)-E_{0}\left(\xi_{0}^{*}\right)\right) \leq\left(E_{T}\left(\xi_{0}\right)-\gamma_{0}\right)^{+} .
$$

Calculating the expectations with respect to $\mathbb{Q}^{*}$ on both sides and multiplying both sides by $\pi$, we obtain

$$
\begin{gathered}
\pi \mathbb{E}^{\mathbb{Q}^{*}}\left(\left(E_{T}\left(\xi_{0}^{*}\right)-\gamma_{0}\right)^{+}\right)+\pi \mathbb{E}^{\mathbb{Q}^{*}}\left(1_{\left\{E_{T}\left(\xi_{0}^{*}\right)-\gamma_{0} \geq 0\right\}}\right)\left(E_{0}\left(\xi_{0}\right)-E_{0}\left(\xi_{0}^{*}\right)\right) \leq \\
\leq \pi \mathbb{E}^{\mathbb{Q}^{*}}\left(\left(E_{T}\left(\xi_{0}\right)-\gamma_{0}\right)^{+}\right) .
\end{gathered}
$$

Using the martingale property and the digital terminal value of the equilibrium allowance prices shown in Proposition 2, we finally obtain

$$
\pi \mathbb{E}^{\mathbb{Q}^{*}}\left(\left(E_{T}\left(\xi_{0}^{*}\right)-\gamma_{0}\right)^{+}\right)+A_{0}^{*}\left(E_{0}\left(\xi_{0}\right)-E_{0}\left(\xi_{0}^{*}\right)\right) \leq \pi \mathbb{E}^{\mathbb{Q}^{*}}\left(\left(E_{T}\left(\xi_{0}\right)-\gamma_{0}\right)^{+}\right) .
$$


For the case that the strategy $\xi_{0}$ yields at least the total production volume of the equilibrium strategy, $V_{0}\left(\xi_{0}\right) \geq V_{0}\left(\xi_{0}^{*}\right)$, assertion (11) in Proposition 1 yields the estimate

$$
C_{0}\left(\xi_{0}^{*}\right)+A_{0}^{*} E_{0}\left(\xi_{0}^{*}\right) \leq C_{0}\left(\xi_{0}\right)+A_{0}^{*} E_{0}\left(\xi_{0}\right)
$$

which is equivalent to

$$
C_{0}\left(\xi_{0}^{*}\right)-C_{0}\left(\xi_{0}\right) \leq A_{0}^{*}\left(E_{0}\left(\xi_{0}\right)-E_{0}\left(\xi_{0}^{*}\right)\right)
$$

Now, combining the last inequality with (23), we obtain

$$
C_{0}\left(\xi_{0}^{*}\right)+\pi \mathbb{E}^{\mathbb{Q}^{*}}\left(\left(E_{T}\left(\xi_{0}^{*}\right)-\gamma_{0}\right)^{+}\right) \leq C_{0}\left(\xi_{0}\right)+\pi \mathbb{E}^{\mathbb{Q}^{*}}\left(\left(E_{T}\left(\xi_{0}\right)-\gamma_{0}\right)^{+}\right),
$$

which proves our claim (22).

In Proposition 3, the equilibrium production schedule $\xi_{0}^{*}$ was characterized as a solution to the minimization problem

$$
\min \left\{\mathbb{E}^{\mathbb{Q}^{*}}\left(B\left(\xi_{0}\right)\right): \xi_{0} \in \times_{i \in I} \Xi^{i}, \quad V_{0}\left(\xi_{0}\right) \geq D_{0}\right\}
$$

Although this fact is about minimization of social burden, it should not be interpreted as one of the classical welfare results, which typically follow from equilibrium considerations.

An interesting point here is that this type of cost-optimality needs to be taken with great care: due to the opportunity cost-pass-through, the consumers can not expect that an (inappropriately designed) cap-and-trade mechanism indeed implements the cheapest way of emission reduction, from their perspective.

To see this point, remember that the price per unit of electricity under the merit order system includes the opportunity costs of consuming the emission certificates. Therefore, given emission price $A_{0}$ and an overall production schedule $\xi_{0}$, the consumers pay the costs $\sum_{i \in I} A_{0} E^{i}\left(\xi_{0}^{i}\right)$ to switch in the merit order and to reduce emissions. From a global perspective, this costs stands for a wealth re-distribution. From the consumer's perspective, it is associated with a burden.

\section{Equilibrium-Like Risk-Neutral Modeling}

Another interesting observation from Proposition 3 is that the expectation $\mathbb{E}^{\mathbb{Q}^{*}}\left(B\left(\xi_{0}\right)\right)$ of the social burden $B\left(\xi_{0}\right)$ is minimized with respect to a risk-neutral measure $\mathbb{Q}^{*}$ which differs from the objective measure $\mathbb{P}$. The measure $\mathbb{Q}^{*}$ is an outcome of the equilibrium, and, as such, it heavily depends on the many model components, for instance on the risk-aversions, on the certificate endowments, and on the production technologies of the agents. However, it is surprising that, once the 
measure $\mathbb{Q}^{*}$ is known, other important equilibrium outcomes can be deduced from aggregated quantities only.

In particular, given $\mathbb{Q}^{*}$, the equilibrium production schedule $\xi_{0}^{*}$ can be obtained as the solution of optimization problem (24). Such solution is determined by aggregated quantities, since the social burden is by definition $B\left(\xi_{0}\right)=C_{0}\left(\xi_{0}\right)+\pi\left(E_{T}\left(\xi_{0}\right)-\right.$ $\left.\gamma_{0}\right)^{+}$and, apart the quantities $\gamma_{0}$ and $\pi$ decided by the authority, it depends only on technologies present in the market. Having obtained the equilibrium production schedule $\xi_{0}^{*}$ as the solution of optimization problem (24), the equilibrium allowance price $A_{0}^{*}$ is calculated applying martingale pricing:

$$
A_{0}^{*}=\pi \mathbb{E}^{\mathbb{Q}^{*}}\left(1_{\left\{E_{T}\left(\xi_{0}\right)-\gamma_{0} \geq 0\right\}}\right) .
$$

Finally, given the production schedule $\xi_{0}^{*}$ and the allowance price $A_{0}^{*}$, also the electricity price $P_{0}^{*}$ is determined as the marginal price of the most expensive technology, which is active in the schedule $\xi_{0}^{*}$. Note that the opportunity costs must be included when identifying the most expensive active technology.

Summarizing, we conclude that given $\mathbb{Q}^{*}$, merely aggregated market parameters are needed to obtain $\xi_{0}^{*}, A_{0}^{*}$, and $P_{0}^{*}$. This observation can be used to establish and to analyze realistic equilibrium-like emission market models. Such models are needed, since in real emission trading it is nearly impossible to estimate the equilibrium from a market model, because the individual parameters are highly undetermined. For instance, within the EU ETS, there are more than 25, 000 agents, each with a specific production, its own certificate endowment, and a completely unknown risk-aversion. On the contrary, the aggregated quantities are well-known, since high-quality market data on total allowance allocation and electricity production, including capacities, costs, and emission rates, are available.

In view of this, we suggest an alternative way to estimate the market equilibrium based on aggregated quantities and using an exogenously specified proxy for risk-neutral measure $\mathbb{Q}^{*}$. This general approach follows the standard methodology of financial mathematics, which successfully describes the stochastic evolution of equilibrium prices on financial markets under an appropriately chosen risk-neutral measure.

\subsection{Market Equilibrium Under a Risk-Neutral Measure}

We sketch the following program for equilibrium-type modeling of emission markets: (1) Determine a risk-neutral measure $\mathbb{Q}^{*}$, which corresponds to an equilibrium situation of the emission market in the sense of (i) of Proposition 2.

(2) Observe that, because of Proposition 3, the equilibrium production schedule $\xi_{0}^{*}$ must be a solution to the deterministic optimization problem 


$$
\begin{aligned}
& \text { minimize } C_{0}\left(\xi_{0}\right)+\pi \mathbb{E}^{\mathbb{Q}^{*}}\left(\left(E_{T}\left(\xi_{0}\right)-\gamma_{0}\right)^{+}\right) \\
& \text {subject to } V_{0}\left(\xi_{0}\right) \geq D_{0}, \text { over } \xi_{0} \in \times_{i \in I} \Xi^{i} .
\end{aligned}
$$

To address the problem further, a specification of the space $\times_{i \in I} \Xi^{i}$ of market production strategies along with the functions $C_{0}, V_{0}$ and $E_{T}$ is required.

(3) Given the equilibrium production schedule, calculate the total production costs $C_{0}\left(\xi_{0}^{*}\right)$, the total carbon dioxide emission $E_{T}\left(\xi_{0}^{*}\right)$, and the energy price $P^{*}$ to assess the performance in emission reduction of the current market architecture.

Remark Note that a risk neutral measure is not unique. Clearly, finding a realistic candidate for the risk-neutral measure $\mathbb{Q}^{*}$ can be difficult. However, notice that one merely needs to specify the fluctuations of the non-predictable emissions under a risk-neutral measure. This distribution can be described in a parameter-dependent way, which adds flexibility to the model. For instance, having assumed a Gaussian framework under objective measure and modeling the density of the risk-neutral measure in terms of a Girsanov kernel. Given theoretical initial emission price depending on the parameters of the Girsanov kernel, these parameters shall be adjusted to match the observed emission prices. Similar techniques have been applied in financial modeling under the framework of implicit model calibration. Being one of the central questions in quantitative finance, the connection between risk-neutral and objective measures has been successfully addressed over the recent decades. In view of this development, modeling from a risk-neutral measure perspective can be based on a variety of different methods, ranging from benchmark approach, estimation of risk premia, state price density from portfolio optimization theory, to several econometric methods for the estimation of the so-called market price of risk.

Finally, the performance of the cap-and-trade mechanism can be examined leveraging on the dependence of the major economic indicators, i.e. total consumers' costs $P_{0}^{*} D_{0}$, total (producers') production costs $C_{0}\left(\xi_{0}^{i *}\right)$, and total carbon dioxide emission $E_{0}\left(\xi_{0}^{i *}\right)$, on the controls available to the regulator.

Note that in the standard scheme the regulator controls two key parameters: the total allowance allocation $\gamma_{0}$ and the penalty size $\pi$. The performance of regulation could be assessed in terms of relation between the increase of consumers' costs versus the achieved emission reduction. Such analysis may uncover and visualize inappropriate market architectures, where unlucky choices of $\gamma_{0}$ and $\pi$ cause consumers to pay too much, compared to emission savings. Complementary or supplementary policies can be evaluated at this point next to the cap-and-trade system. In particular, different forms of subsidies and carbon tax mechanisms can have a strong impact on the merit order of different technologies.

\section{Conclusions}

In this paper, we show how equilibrium analysis and optimization of an environmental market can be carried out under the realistic assumption of risk-averse market players. 
This generalization is based on a novel approach. Thereby, we obtain a number of interesting observations, which allow studying equilibrium market situations in terms of aggregated market quantities under a risk-neutral measure. Our findings show how market design optimization can be achieved incorporating risk-aversion. The choice to develop our approach in one-period setting, yields explicit results which constructively contribute to better understand the working principles of financial instruments and to improve both effectiveness and efficiency of environmental policy.

Open Access This chapter is distributed under the terms of the Creative Commons Attribution Noncommercial License, which permits any noncommercial use, distribution, and reproduction in any medium, provided the original author(s) and source are credited.

\section{References}

1. Barrieu, P., Fehr, M.: Integrated EUA and CER price modeling and application for spread option pricing. Centre for Climate Change Economics and Policy Working Papers 50, Centre for Climate Change Economics and Policy, London, UK (2011)

2. Carmona, R., Fehr, M.: The clean development mechanism and joint price formation for allowances and CERs. In: Dalang, R., Dozzi, M., Russo, F. (eds.) Seminar on Stochastic Analysis, Random Fields and Applications VI. Progress in Probability, vol. 63, pp. 341-383. Springer, Basel (2011)

3. Carmona, R., Fehr, M., Hinz, J.: Optimal stochastic control and carbon price formation. SIAM J. Control Optim. 48(4), 2168-2190 (2009)

4. Carmona, R., Fehr, M., Hinz, J., Porchet, A.: Market design for emission trading schemes. SIAM Rev. 52(3), 403-452 (2010)

5. Chesney, M., Taschini, L.: The endogenous price dynamics of the emission allowances and an application to $\mathrm{CO}_{2}$ option pricing. Swiss Finance Institute Research Papers 08-02, Swiss Finance Institute, Zurich, Switzerland (2008)

6. Dales, J.H.: Land, water, and ownership. Can. J. Econ. 1(4), 791-804 (1968)

7. Hinz, J., Novikov, A.: On fair pricing of emission-related derivatives. Bernoulli 16(4), 1240$1261(2010)$

8. Kabanov, Y., Sticker, C.: A teachers' note on no-arbitrage criteria. In: Azéma, J., Émery, M., Ledoux, M., Yor, M. (eds.) Séminaire de Probabilités XXXV. Lecture Notes in Mathematics, vol. 1755, pp. 149-152. Springer, Berlin (2001)

9. Kijima, M., Maeda, A., Nishide, K.: Equilibrium pricing of contingent claims in tradable permit markets. J. Futures Mark. 30(6), 559-589 (2010)

10. Montgomery, W.D.: Markets in licenses and efficient pollution control programs. J. Econ. Theory 5(3), 395-418 (1972)

11. Seifert, J., Uhrig-Homburg, M., Wagner, M.: Dynamic behavior of $\mathrm{CO}_{2}$ spot prices. J. Environ. Econ. Manage. 56(2), 180-194 (2008)

12. Sijm, J., Neuhoff, K., Chen, Y.: CO2 cost pass-through and windfall profits in the power sector. Clim. Policy 6, 49-72 (2006)

13. Stevens, B., Rose, A.: A dynamic analysis of the marketable permits approach to global warming policy: a comparison of spatial and temporal flexibility. J. Environ. Econ. Manage. 44(1), 45-69 (2002)

14. Taschini, L.: Environmental economics and modeling marketable permits. Asia-Pac. Finan. Mark. 17(4), 325-343 (2010) 